\title{
Visión General, Características y Funcionalidades de la Red Eléctrica Inteligente (Smart Grid)
}

\author{
Víctor A. Gómez ${ }^{(1)}$, Cesar Hernández ${ }^{(1) \star}$ y Edwin Rivas ${ }^{(2)}$ \\ (1) Facultad Tecnológica, Universidad Distrital Francisco José de Caldas, Bogotá-Colombia. \\ (e-mail: victorgomez8807@gmail.com; cahernandezs@udistrital.edu.co) \\ (2) Facultad de Ingeniería, Universidad Distrital Francisco José de Caldas, Bogotá-Colombia. \\ (e-mail: erivas@udistrital.edu.co)
}

* Autor a quien debe ser dirigida la correspondencia

Recibido Jul. 28, 2017; Aceptado Oct. 6, 2017; Versión final Nov. 19, 2017, Publicado Abr. 2018

\begin{abstract}
Resumen
Este artículo presenta una visión general de la red eléctrica inteligente (Smart Grid) con sus características y funcionalidades y se identifica las actividades de investigación, tendencias, problemas y desafíos de la Smart Grid, Para ello se realizó una revisión de la literatura donde se visualiza como la integración de los recursos energéticos distribuidos (generación distribuida y almacenamiento energético), así como los avances de las tecnologías de la información y las comunicaciones, han desarrollado el concepto de la moderna red eléctrica. En esta área, la implementación de políticas y la rápida integración de sistemas de gestión, están teniendo como resultado una gran revolución en la generación, la trasmisión, la distribución, la operación y el consumo energético.
\end{abstract}

Palabras clave: red eléctrica inteligente; recursos energéticos distribuidos; micro-redes; redes eléctricas inteligentes

\section{Overview, Features and Functionalities of the Smart Grid}

\begin{abstract}
This paper presents an overview of the smart grid with its features and functionalities and identifies the research activities, developments, problems and challenges of smart grids. To achieve this objective, a literature review was done, finding how the dynamic integration of developments in electrical engineering, energy storage and advances in information and communication technologies has shaped the concept of the modern electricity network. In this area, policy implementation and the rapid integration of management systems are causing a great revolution in the generation, transmission, distribution, operation and energy consumption.
\end{abstract}




\section{INTRODUCCIÓN}

Las redes inteligentes también conocidas como Smart Grid (SG), surgieron como una respuesta a la necesidad de modernizar la red eléctrica, articulando los procesos de control y monitoreo con tecnologías verdes, también conocidas como no contaminantes o ecológicas. Las redes inteligentes permiten que los recursos energéticos distribuidos, por sus siglas en ingles DER se conecten de forma segura a la red (Keyhani, 2016). Las redes inteligentes son autónomas y mejoran la eficacia y eficiencia en la gestión de energía eléctrica, permitiendo que las empresas de servicios públicos optimicen la infraestructura existente, minimizando la construcción de más plantas de energía (Kobus et al. 2015).

Este artículo identifica las tendencias, problemas y los desafíos de la SG, en primera instancia presenta una visión general de la SG con sus características y funcionalidades, ratifica como cómo la tecnología ha configurado la moderna red eléctrica (Restrepo-Garcés, Manotas-Duque, y Lozano, 2016). Igualmente, se analizan las políticas, proyectos piloto más representativos y sus implementaciones.

Por ende surge un interrogante: ¿Qué es Smart Grid? Donde Smart significa inteligente, que opera en la automatización; Grid, se refiere a la red eléctrica; una red de líneas de transmisión, subestaciones, transformadores y de más elementos que entregan electricidad desde la planta de energía a los usuarios finales. Al igual que el Internet, Smart Grid consistirá en sistemas de control, ordenadores, comunicación y nuevas tecnologías y equipos trabajando en conjunto, pero en este caso, estas tecnologías funcionarán con la red eléctrica para responder digitalmente a nuestra demanda eléctrica. Consecuentemente, esta moderna red es capaz de almacenar, comunicar y tomar decisiones. La Fig. 1 ilustra de manera didáctica la estructura de la SG.

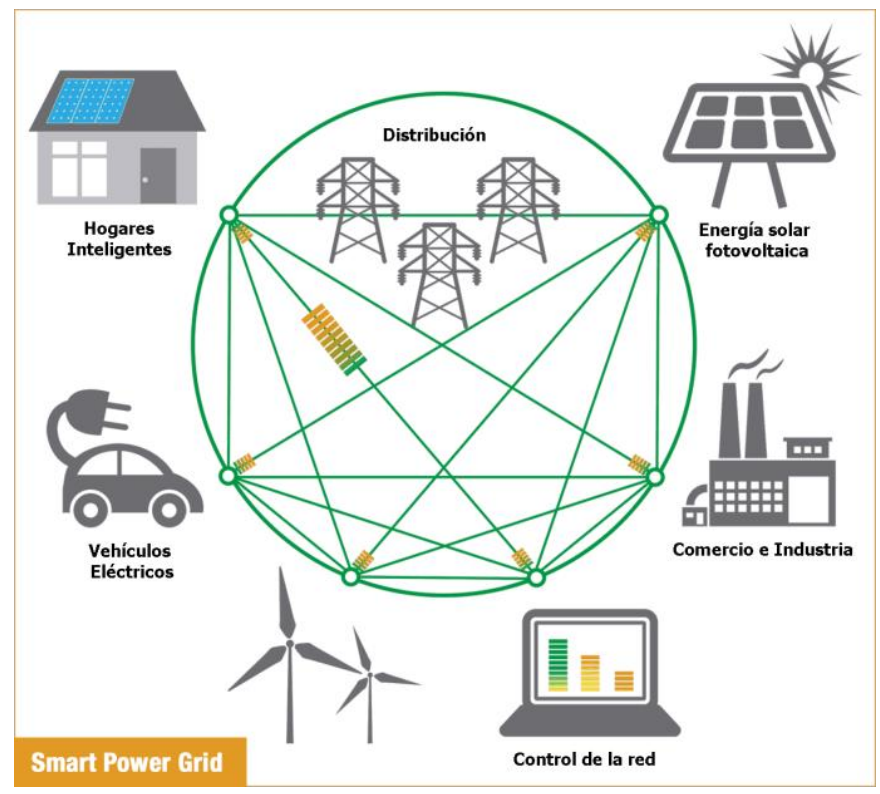

Fig. 1: Estructura de la Smart Grid (R. Abby, 2014)

Otras definiciones indican que una SG es una red eléctrica que puede integrar inteligentemente las acciones de todos los usuarios generadores y consumidores conectados a ella, y aquellos que hacen ambas cosas para conseguir de manera eficiente, económica y segura la electricidad. Los planes de expansión y modernización, tales como: SG de Corea 2030 indican que una SG se refiere a una red de próxima generación que integra la Tecnología de la Información (TI) en la red eléctrica existente, y de esta manera, se brinda una optimización en la eficiencia energética a través de un intercambio bidireccional de información eléctrica entre proveedores y consumidores en tiempo real (Mah et al. 2012).

Según el Instituto Nacional de Estándares y Tecnología (NIST), las SG son un sistema de cuadrícula que integra muchas variedades de tecnologías y servicios de computación y comunicación digital en la infraestructura del sistema eléctrico. Por lo tanto las SG van más allá de los medidores de energía inteligentes para hogares y empresas, ya que los flujos bidireccionales de energía y las capacidades bidireccionales de comunicación y control pueden aportar nuevas funcionalidades. En otras palabras, las posibilidades de la SG son vastas en los grandes contrastes de la tecnología moderna y el aumento de la interdependencia entre los actores del sector eléctrico. A manera de ejemplo, las SG pueden proporcionar una plataforma para maximizar la confiabilidad, disponibilidad, eficiencia, desempeño económico y la mayor 
seguridad frente a los ataques y las interrupciones de energías naturales. La SG puede entenderse mejor cuando se ve junto a la red tradicional. Es así como en Yu et al. (2012) se detalla una buena comparación entre las dos estructuras (red tradicional vs SG).

La infraestructura o el diseño de un sistema SG siempre están relacionados con los objetivos y capacidades establecidas. A manera de ejemplo, la implementación de una SG puede mejorar la robustez, la capacidad de auto recuperación y la integralidad de la red. Es así como NIST presenta un modelo conceptual que apoya la planificación, el desarrollo de requisitos, la documentación y la organización de redes y equipos interconectados que componen la SG. Por esta razón, NIST ha dividido la SG en siete dominios (con subdominios) que abarcan a los actores y aplicaciones SG. Además, clasifica como dispositivos actores (medidores inteligentes y generadores de energía solar), sistemas de control, programas y grupos de interés que toman decisiones e intercambian información necesaria para realizar aplicaciones. Estas últimas como aplicaciones de tareas realizadas por uno o más actores dentro de un dominio (domótica, generación de energía solar, almacenamiento de energía y gestión de la energía).

\section{SG Y SU FUNCIONALIDAD}

La SG propone respuestas y soluciones a las preocupaciones de adecuación del suministro de electricidad. Es por ello que numerosos países, han preparado el terreno para la modernización de la red eléctrica, a través de la legislación, subsidios, asesoramiento para el ahorro de energía, tratados internacionales, técnicas de políticas públicas e incentivos a la inversión. Un ejemplo de lo anterior se evidencia en Estados Unidos y China, quienes han realizado grandes esfuerzos en la construcción de una red inteligente como una sustitución de la red energética tradicional de uso intensivo de energía (Wu, Chen, y Liu, 2011).

Estos grandes retos trazados por los países desarrollados han permitido que en las siguientes secciones se aprecie de manera general las funcionalidades que deben cubrir el desarrollo e implementación de las redes inteligentes.

\section{Estandar IEEE 2030}

Desde el punto de vista normativo la IEEE Std 2030 ha sido desarrollada con el objetivo de proporcionar enfoques alternativos y mejores prácticas para lograr la interoperabilidad de la red inteligente. Éste estándar ha trazado la hoja de ruta dirigida a establecer el marco en el desarrollo de un cuerpo de normas nacionales e internacionales, basado en técnicas transversales en aplicaciones de potencia e intercambio y control de la información mediante comunicaciones. En este orden de ideas, este estándar ha definido tres perspectivas arquitectónicas integradas: sistemas de energía, tecnología de comunicaciones y tecnología de la información; las cuales indican las principales directrices para la interoperabilidad de la Smart Grid.

\section{Fiabilidad, seguridad y eficiencia de la red eléctrica}

Adaptar de los estándares internacionales la interoperabilidad de la red eléctrica, permite tener una filosofía orientada a la confiabilidad del suministro, más aun cuando la continuidad en la fuente de alimentación es crucial para cualquier sistema de energía (Hernandez, Gomez, y Pena, 2014). El éxito de la red es proporcionar el servicio necesario a los usuarios finales con calidad y confiabilidad en el suministro, mejorando la detección y permitiendo la auto restauración del sistema (Xia et al. 2014). A medida que las redes continúan creciendo en tamaño y complejidad, se hace más difícil analizar la confiabilidad de la red, pero los nuevos métodos analíticos de los esfuerzos de investigación han continuado construyendo una de las bases de análisis y control para redes más confiables. Por ejemplo, un algoritmo de minería de datos puede descubrir la estructura del sistema a partir de datos crudos e históricos para poder estimar la fiabilidad del servicio de la red utilizando redes bayesianas (Doguc y Ramirez-Marquez, 2012).

Por otro lado, el monitoreo remoto de la generación híbrida y la gestión automática de SG para distribución inestable contribuye a la eficiencia principal. La red de información en SG permite muchas características y, aunque propenso a los ataques, ha sido contrarrestada por soluciones prometedoras, tales como los sistema de detección de intrusiones (IDS) (T. Liu et al. 2015) o por ocultamiento de la información confidencial dentro de las lecturas normales usando Wavelet Steganographic (Di Tommaso et al. 2010).

El análisis de amenazas en sistemas SG y el modelo de amenazas de seguridad en sistemas integrados, ayuda a comprender mejor las debilidades explotadas por los atacantes (Suleiman et al., 2015). Por ejemplo, el enfoque de la teoría de juegos, basado en interacciones en estructuras formalizadas de incentivos, permite llevar a cabo procesos de decisión para abordar el tema de la seguridad cibernética en el monitoreo y la protección. Igualmente, el control desde una perspectiva coordinada ciber-ataque (Ashok et 
al. 2014) puede mejorar la seguridad. En resumen, las asociaciones del sector de la energía están gestionando la ciberseguridad al tiempo que mantienen las funciones críticas de suministro de energía para garantizar la fiabilidad de la red modernizada.

Sin embargo, los mayores aportes a la fiabilidad, la seguridad y la eficiencia de la red eléctrica han tenido lugar en el desarrollo de algoritmos inteligentes de optimización tales como: algoritmos genéticos, redes neuronales, estrategias de teoría de juegos, aprendizaje por refuerzo, razonamiento basado en casos, máquinas de soporte vectorial, entre otras. Las anteriores estrategias, han permitiendo estudiar las interacciones en estructuras formalizadas de la seguridad ante la respuesta a la demanda en los mercados energéticos. Por consiguiente, los sistemas modernos de control y monitoreo de las SG han realizado una rápida identificación de los elementos clave en la infraestructura. Por ejemplo, Jarmakiewicz, Parobczak, y Maślanka (2017) detallan una estrategia de protección estudiando los principales puntos vulnerables de la red eléctrica para luego implementar algoritmos de optimización.

\section{Implementación e integración de recursos distribuidos y generación}

Los Recursos Energéticos Distribuidos (DER) son pequeñas fuentes de energía que pueden ayudar a satisfacer la demanda de energía regular. Es así como las DER con tecnologías de almacenamiento y con tecnologías renovables facilitan la transición a SG (Birk, 2016). La llegada de fuentes de energía renovable como generadores distribuidos, puede ayudar a mitigar los problemas de agotamiento de las reservas de combustibles fósil. Así como tener una oportuna respuesta a la creciente demanda de los consumidores. Es así como la generación distribuida que incluye generadores eólicos, generadores fotovoltaicos y sistemas de almacenamiento de baterías, puede incorporar generación térmica y vehículos eléctricos. Sin embargo, la inclusión de estas fuentes, también significa que cantidades innumerables de datos necesitarían ser manejados y procesados. Por ejemplo, la investigación desarrollada en Penya et al. (2012) presenta una arquitectura de nodos inteligentes individuales que controlan una serie de activos eléctricos a partir del procesamiento de datos registrados por las redes de sensores inteligentes.

\section{Respuesta a la demanda y recursos de la demanda}

La Comisión Federal de Regulación de la Energía define la respuesta a la demanda como: "Los cambios en el consumo de energía de los clientes o subscriptores del servicio eléctrico para adaptar mejor la demanda de energía con el suministro" (Rahimi y Ipakchi, 2010). En concordancia, La respuesta a la demanda proporciona a los consumidores, la oportunidad de participar en las operaciones de la red, ya que pueden reducir o cambiar los perfiles de consumo durante los períodos de máxima actividad y beneficiarse mediante incentivos financieros. El desarrollo de tecnologías y técnicas de modernización de la red para la respuesta a la demanda, es uno de los objetivos principales de los países desarrollados. En donde, los recursos de la demanda o los programas de eficiencia energética y de la gestión de la carga, cada vez más son impulsores de las crecientes inversiones que incluyen preocupaciones ambientales, económicas y de fiabilidad.

\section{Automatización de la medición de energía}

La medición en SG permite la comunicación bidireccional entre los medidores, el usuario y el operador de red. Los medidores aseguran facturas más precisas y ponen a los consumidores en un papel protagónico en la cadena de valor energética. Los medidores inteligentes, como normalmente se llaman, implican sensores, notificación de corte de energía y control de calidad de la potencia.

La automatización en la cadena de valor energética siempre está asociada con los medidores inteligentes. Es decir, con la Infraestructura de Medición Avanzada (IMA), las empresas de servicios públicos pueden recopilar información de los consumidores más rápidamente. Es así como es posible proporcionar una red de comunicaciones de todo el sistema a puntos de servicios públicos y dispositivos de enlace a través de la red. El IMA y la automatización de la distribución abren la puerta a una gran modernización de la red a través de la monitorización de transformadores y alimentadores, la gestión de interrupciones, la integración de vehículos eléctricos y el aislamiento eficaz de fallas. Una forma de lograr la automatización de la distribución es a través de la implementación del sistema de automatización para subestaciones, este sistema define las acciones de control local para resolver la congestión con una reducción mínima de las fuentes de energía renovable (Berizzi et al. 2015).

\section{Integración de dispositivos inteligentes}

Los aparatos y dispositivos inteligentes son piezas de equipo que pueden comunicarse con las redes eléctricas, apagarse durante las horas pico de consumo y son capaces de cambiar el uso inteligente de la 
energía por sí mismos. Por ejemplo, en un estudio realizado en Gran Bretaña, la respuesta a la demanda en los hogares con una penetración del $20 \%$ de los aparatos inteligentes puede proporcionar hasta un $54 \%$ de los requisitos de reservas operativas en función del momento del día (Nistor et al. 2015). De esta manera, los aparatos inteligentes cambian la demanda de electricidad de los hogares aumentando así aspectos importantes tales como: el uso racional de la energía y la eficiencia eléctrica.

Un estudio sobre los hogares que utilizan la automatización de la lavadora inteligente ha visto que la demanda se desplazó automáticamente a períodos de tiempo en los que la oferta de electricidad es abundante (Kobus et al. 2015). Lo anterior fue posible con la implementación de una red de área doméstica de sensores inalámbricos que utiliza el protocolo ZigBee, empleado para retransmitir mensajes entre diferentes entidades en un esquema de gestión de la energía basado en la coordinación de electrodomésticos.

\section{Tecnologías avanzadas de almacenamiento de electricidad}

El almacenamiento de electricidad y las tecnologías que intentan moderar y reducir picos en el perfil de carga de los usuarios es una funcionalidad esencial de la SG. El almacenamiento de energía es indispensable porque la generación de electricidad a partir de energías renovables fluctúa. Los dispositivos de almacenamiento, almacenan el excedente de electricidad cuando la generación de energía renovable es abundante, por lo que el sistema puede utilizar esta energía a medida que aumenta la demanda. Los Vehículos Eléctricos (VE) pueden servir a la red eléctrica como fuente de energía independiente. Pueden permanecer conectados a la red una vez que están estacionados, y así entregar la energía de sus baterías en una tecnología conocida como Vehículo a la Red (V2G).

\section{Opción oportuna de información y control}

La información oportuna sobre la red eléctrica se logra cuando estas se hacen más inteligentes (Ver Fig. 2). La generación, la transmisión, la distribución y los consumidores deben tomar decisiones informadas en el momento más apropiado. La sincronización de tiempo y los dispositivos inteligentes de punto final permiten la recopilación de datos esenciales para una detección más rápida de los consumidores ilegales, la detección de sobrecarga y la verificación de calidad de energía (Stastny et al. 2015). Por otro lado, el control inteligente en SGs es necesario para una programación óptima de las fuentes de energía, para maximizar el transporte de energía, para la estabilidad transitoria y para el control de la potencia real y reactiva. Lo anterior, como mecanismos de optimización en la generación de energía mediante el seguimiento perfecto de las fluctuaciones de la demanda de la carga. Así mismo, el control inteligente puede utilizarse para la detección y reconfiguración autónoma de fallas (Tarhuni et al. 2015).

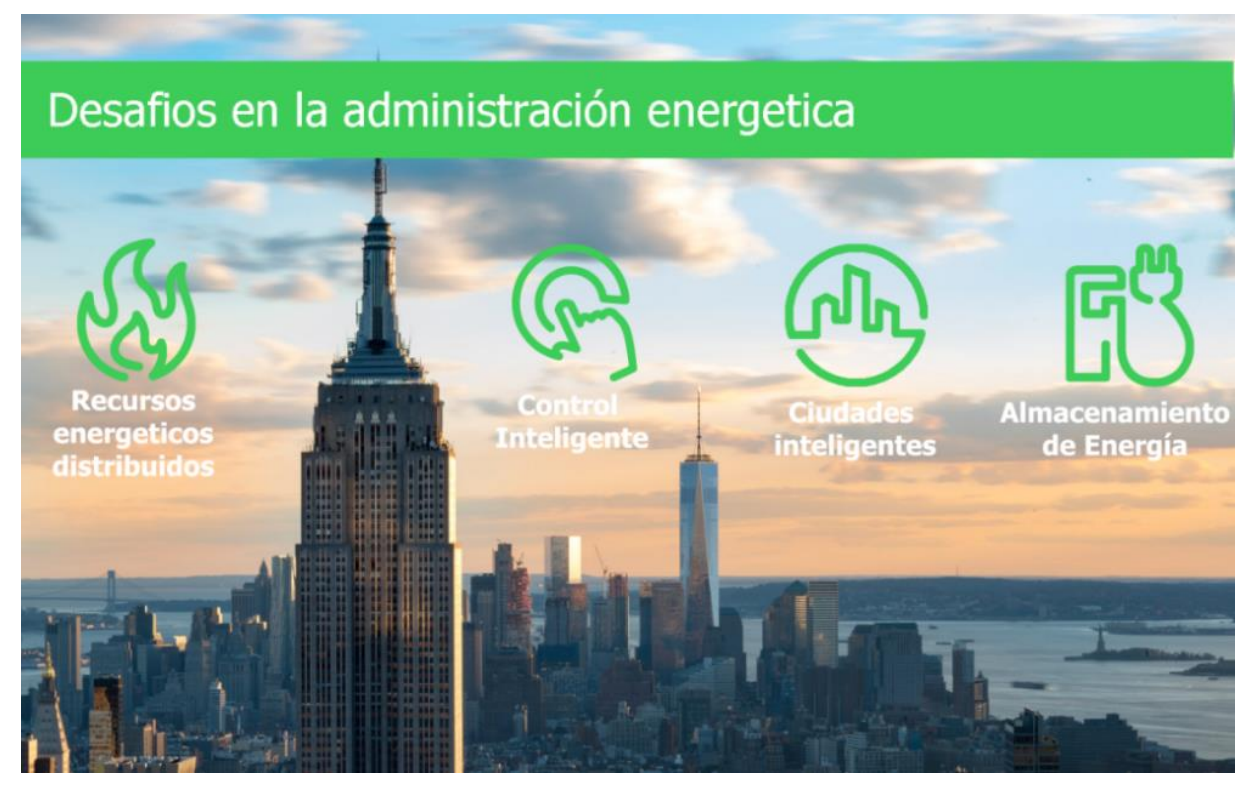

Fig. 2: Control inteligente de la SG (Electric, 2017)

La investigación desarrollada en Reddy et al. (2014) destaca los diferentes métodos de las características importantes de las SG; Integración, Control, Comunicación y Medición (ICCM). Por ejemplo, la implementación de este tipo de metodología proporciona la fecha exacta y hora de los eventos de la red, destacando los eventos de mayor importancia. 
Los componentes de la red deben ser capaces de trabajar juntos para permitir el transporte fiable de la electricidad desde la generación hasta el consumo. La interoperabilidad es crucial en la planificación e implementación de la arquitectura de la red. La complejidad, el número y la escala de los sistemas y dispositivos que participan en las redes inteligentes hacen indispensable este concepto. Es por ello que el Grupo Programador de los Incentivos de Autogeneración (SGIP) como consorcio de la industria y representando una sección trasversal del ecosistema energético, ha iniciado la tarea de coordinar el desarrollo de estándares para SGs y asegurar que los componentes puedan operar y comunicarse perfectamente entre sí. Todos los dominios integrados del sistema eléctrico, los clientes, los mercados, los proveedores de servicios, las operaciones, la generación a granel, la transmisión y la distribución, trabajan juntos para construir una red moderna y eficiente. Algunos de los estudios sobre esta área han examinado los problemas de interoperabilidad en las redes inalámbricas heterogéneas para las ciudades inteligentes (Avelar et al. 2015). Llegando a la conclusión, de que la interoperabilidad será el elemento clave en el diseño y operación de la nueva red eléctrica confiable y flexible.

\section{TECNOLOGÍAS Y ACTIVIDADES DE INVESTIGACIÓN}

En este apartado se mencionan algunas de las principales tecnologías de investigación de las SG. Lo anterior, en función de la integración dinámica de los desarrollos de ingeniería eléctrica, almacenamiento energético y los avances de las Tecnologías de la Información y las Comunicaciones (TIC).

\section{Control}

En las áreas de control, las redes inteligentes están utilizando métodos y técnicas de conexión con el precepto de garantizar una red de energía limpia. Estos métodos son: El método de control basado en la electrónica de potencia, el método de control basado en un sistema (MAS), el método avanzado de control de fallas y el método de Suplencia Virtual (VPP) (Peng y Yan, 2011). Este último, son sistemas compuestos por unidades de generación distribuidas de pequeño tamaño que forman unidades de generación virtuales tales como: microCHP, turbinas eólicas, pequeñas centrales hidroeléctricas, unidades fotovoltaicas, grupos electrógenos de respaldo y baterías. Estas unidades pueden ser manejadas individualmente integradas o no a una red centralizada.

Con la aparición de dispositivos electrónicos de potencia en algunas de las tecnologías y/o técnicas descritas con anterioridad, las grandes centrales eléctricas convencionales han logrado integrarse en el sistema de red utilizando pequeños Sistemas de Conversión de Energía (ECS). Estos sistemas de conversión son capaces de operar autónomamente, conectados a la red o aislados. Existen varias configuraciones de topologías de conversión de pequeña y mediana potencia, incluyendo su control. Por ejemplo, la configuración de la turbina eólica y su respectivo operación a través de sistemas Multiagente (MAS), cuya función específica resulta en realizar un control inteligente para variables con persistentes cambios en la frecuencia de la carga (Singh, et al., 2017).

Sin embargo, los ECS existentes no pueden manejar un gran número de componentes renovables en los sistemas de energía limpia. Es por ello que los sistemas MAS están siendo fielmente utilizados para suplir estas necesidades de control del suministro energético. El uso de MAS para controlar una red distribuida de la SG demostró que el mismo permite una transición sin problemas desde la red conectada a un modo isla en caso de que se detecten interrupciones en algún punto de la instalación. Por lo tanto, un sistema MAS es una tecnología útil para la gestión de las MG. La gestión avanzada de fallas es posible gracias a la coordinación completa de la automatización local. Es por ello que estos sofisticados sistemas se puede utilizar para proteger a los consumidores importantes de interrupciones. En este orden de ideas, Las técnicas diagnósticas son importantes en MG y en la SG, ya que deben ser tolerantes a fallos. Una estrategia de control en tiempo real para la central eléctrica virtual podría cubrir la fluctuación de la producción de generación distribuida no controlable y la carga mutacional sin necesidad de establecer una medición constataste. (Yuan et al. 2014).

Otras investigaciones en el área de control son: el manejo óptimo del servicio de control de generación automática en las redes de usuarios inteligentes, incluyendo los vehículos eléctricos y los recursos distribuidos; el esquema de control de carga dinámica para las SG; arquitectura de control de la formación de islas de generación hacia el futuro; la SG con la demanda y la turbina de viento; control inteligente de amenazas operativas en subestaciones; generador de energía eólica para aplicaciones de SG utilizando control de potencia neuro-fusible codificado inalámbricamente y efectos de comunicación en el control de frecuencia (Kilkki et al., 2014). 
Igualmente, los estudios relacionados en las áreas de las comunicaciones incluyen incluyen: requisitos de red de comunicación para las principales aplicaciones de SG en redes aéreas domesticas (HAN), en redes aéreas cercanas (NAN) y en redes aéreas amplias (WAN); comunicación resistente para la red de sensores ubicua de SG; nuevos algoritmos de enrutamiento multipath para comunicaciones tolerantes a fallos en SG; estrategias para el despliegue inteligente de redes de conmutación de comunicaciones en línea; marco de procesamiento ciego para facilitar la apertura en las comunicaciones de SG; impacto del enrutamiento escalable en la vida de las redes de comunicación SG; evolución de TIC implementadas en las SG (Kuzlu et al., 2014).

\section{Comunicación}

Las redes inteligentes abarcan la integración de las tecnologías de la información para la infraestructura de la red eléctrica. En donde la gestión está dada en el control de equipos y cargas. En consecuencia, la operación automática del sistema, permite opciones productivas tanto en los operadores de servicios públicos como en los clientes. Lo anterior bajo el precepto de garantizar la confiabilidad y continuidad en el suministro eléctrico.

El primer paso para llegar a todas estas experiencias altamente significativas está sustentado en el estándar IEEE Std 2030, como una guía para la interoperabilidad de la red eléctrica inteligente con las tecnologías de la información. Este estándar es una hoja de ruta dirigida a establecer el marco de desarrollo de disciplinas y técnicas transversales en aplicaciones de potencia e intercambio de información y control a través de comunicaciones. Es decir, la interoperabilidad que argumenta este estándar, reside en la capacidad de dos o más redes, sistemas, dispositivos, aplicaciones o componentes para intercambiar externamente y utilizar fácilmente la información de forma segura y efectiva. La interoperabilidad de Smart Grid brinda a las organizaciones la capacidad de comunicarse de manera efectiva y transferir datos significativos, a pesar de que pueden estar usando una variedad de diferentes sistemas de información sobre infraestructuras muy diferentes, a veces en diferentes regiones geográficas(Kaschel Cárcamo y Viera Riquelme, 2017).

El segundo paso para llegar a estas experiencias reside en el término Internet de las Cosas (IoT). Cuyo término fue introducido por primera vez en 1999 por Auto-ID Labs, principalmente para dispositivos de identificación por radiofrecuencia en red (RFID). Desde entonces, el concepto ha evolucionado y hoy en día IoT abarca muchas otras tecnologías, incluidas las redes inalámbricas de sensores (WSN), la biotecnología y redes de área corporal (BAN), comunicaciones máquina a máquina (M2M) y otras como WiFi, Bluetooth, entre otros. En la Fig. 3 se logra apreciar el sin número de tecnologías de la información y las telecomunicaciones que pueden operar en un sistema de distribución eléctrica.

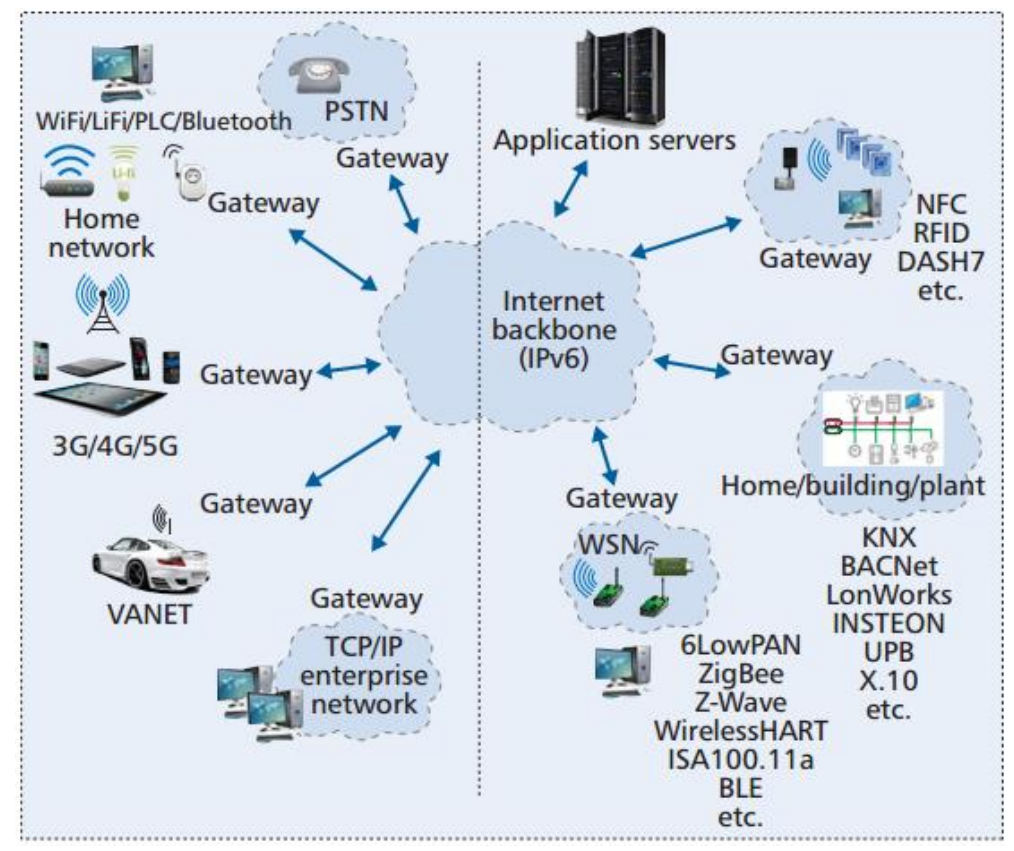

Detección y medición

Fig. 3: Arquitectura loT (Meddeb, 2016)

Los sensores son componentes esenciales de la SG. Estos pequeños nodos sirven como estaciones de detección y permiten el monitoreo remoto de equipos y fuentes de energía. Los sensores de alta velocidad 
llamados síncrofasores o Unidades de Medida del Fasor (PMU) son dispositivos que proporcionan mediciones sincronizadas de fasores en tiempo real de valores de tensión y corriente. Estas mediciones de fasor se utilizan en monitoreo avanzado de sistemas de potencia, aplicaciones de protección y control. Las PMU son 100 veces más rápidas que el Control de Supervisión y Adquisición de Datos (SCADA) y son capaces de registrar las condiciones de la red con gran precisión debido a la implementación de protocolos de comunicación como el estándar IEEE C37.118-2005, (Singh y Grape, 2014)

Por último, algunos estudios que involucran sensores son: comunicación resistente para la red de sensores ubicua de SG utilizando el estándar IEC 61850 para automatización de las subestaciones eléctricas; vigilancia segura y confiable sobre redes cognitivas de sensores de radio en SG; diferenciación de calidad de servicio en ruta única y multi-pista para aplicaciones inteligentes y redes de sensores de radio cognitivas. No obstante, las investigaciones desarrolladas en Tsado et al., (2015); detalla los diferentes sistema de comunicación para las SG.

\section{Vehículos eléctricos VE}

Los VE y las aplicaciones V2G ofrecen muchos beneficios. V2G es una tecnología en la cual los Vehículos Eléctricos Híbridos Enchufables (PHEV) o los vehículos eléctricos con respaldo en combustible, se comunican con la red eléctrica para proporcionar la energía máxima y la reserva que pudiese ofertar a la red. La programación de V2G puede suavizar drásticamente la fluctuación en los perfiles de carga en la demanda energética. En consecuencia, El concepto V2G puede mejorar la eficiencia, la estabilidad y la fiabilidad de la red (Murat Yilmaz y Krein, 2012). Con base en lo anterior, los costos de los propietarios de vehículos se reducen a la mitad con el uso de VE y en términos de pérdidas los resultados resultan ser positivos para los agentes del sector eléctrico. Por otro lado, la comunicación en tiempo real, la medición inteligente y las HAN pueden mejorar la capacidad de V2G para la carga y descarga coordinada de los VE. Por esta razón, un estudio de caso en Portugal muestra una buena correspondencia entre la factura inteligente y los perfiles de producción fotovoltaica (Nunes et al. 2015) en los sistemas de generación distribuida. Por consiguiente, la carga es una parte esencial de la tecnología V2G y los trabajos relacionados con la carga y descarga han sido dinámicos, sobre los cuales hay un gran número de documentos y procedimientos.

Otras investigaciones que involucran este conjunto de tecnologías son: Los VE híbridos enchufables y las SG; el impacto armónico de varios vehículos eléctricos asumiendo una carga rápida sobre el sistema eléctrico centralizado; los VE que interactúan con energía renovable; el sistema de gestión de energía inteligente adaptable a VE híbrido enchufable; integración de vehículos eléctricos híbridos enchufables en estructuras existentes del sistema eléctrico; análisis de impacto de las tecnología de VE en la red; estrategias de carga de VE en redes de distribución; programación óptima de los VE en un estacionamiento inteligente; la integración de la red de fuentes de energía renovables intermitentes utilizando VE enchufables que responden a los precios y el análisis del ciclo de vida de la infraestructura de suministro de energía para los vehículos convencionales y eléctricos (Lucas et al., 2012).

\section{Seguridad en SG}

La seguridad de la SG es una ramificación de las complejas redes compuestas por millones de dispositivos y entidades que se conectan entre sí. Las vulnerabilidades en las rede inteligente es más común en medidores inteligentes, dispositivos que interactúan con la oferta y la demanda de electricidad. Lo anterior, también está en función de la ubicación geográfica en donde se instalan los medidores y del nivel de encriptación con lo cual son codificados los algoritmos de análisis del consumo energético. Por ejemplo, Bekara (2014) investigó los problemas y desafíos de la seguridad en las redes inteligentes basadas en el Internet de las cosas (IoT) y definió los principales servicios de seguridad que deberían considerarse. Así pues, las metodologías inmersas en las loT son la evolución al siguiente paso de la Internet de hoy, donde los objetos físicos están equipados con capacidades de computación y comunicación.

Un ejemplo de seguridad en las SG se detalla en Farag et al. (2014), en donde los resultados de la simulación inicial en un estudio sobre el marco de seguridad de capas cruzadas ilustran la factibilidad y efectividad de la capa de seguridad física desarrollada utilizando un modelo conceptual de capas de seguridad. Es decir, la asignación de múltiples barreas de acceso codificado para la posible intromisión en la información registrada por los medidores de energía. Por otro lado, otras investigaciones de seguridad incluyen: seguridad basada en ubicación para aplicaciones de SG; modelo de amenaza de seguridad de sistemas SG integrados; medición inteligente de preservación de la privacidad con múltiples consumidores de datos y mecanismo de privacidad de código utilizando la arquitectura de comunicación en anillo. 


\section{Simuladores y sistemas de información}

Las herramientas computacionales ayudan a los agentes del sector eléctrico, a lograr formas fácilmente comprensibles a partir de datos complejos de gran tamaño. Los sistemas y soluciones de software ofrecen estrategias que permitir la planificación, la coordinación y el funcionamiento seguro de las operaciones de la red. Un marco de co-simulador para sistemas de monitoreo SG de área amplia basado en PMU es presentado en Bhor, Angappan y Sivalingam (2016), a fin de monitorear los eventos desfavorables de la red eléctrica para la posterior toma de decisiones cuyo objetivo sea garantizar la confiabilidad y calidad de la potencia eléctrica. , la simulación de la SG es una herramienta esencial en su diseño e implementación. Las simulaciones permiten una variedad de escenarios y configuraciones. Las plataformas de simulación en tiempo real pueden simular redes inteligentes complejas con muchos dispositivos de conmutación y éstas son eficaces para probar la comunicación y el control distribuido.

\section{Integración de las energías renovables}

Aunque muchos estudios sobre energías renovables se han llevado a cabo para explorar fuentes adicionales de energía limpia, la integración de las dichas fuentes en el sistema eléctrico son uno de los retos en la modernización de la red eléctrica y en el desarrollo de las SG, debido a que algunas redes ya están muy congestionadas y el poder de movimiento de los parques eólicos a la red puede ser difícil. No obstante, las fuentes de energía renovables son intermitentes e inherentemente variables. Tradicionalmente, la electricidad ha fluido de una manera, de una central eléctrica a un cliente; ahora que al implementar fuentes adicionales procedentes de fuentes alternativas, la electricidad tiene que entrar en la red desde múltiples ubicaciones, lo cual demanda una arquitectura tecnológica basada en: la automatización de la red y un óptimo flujo de energía bidireccional. Es así como los controles modernos son necesarios para llevar viento, energía solar y otras fuentes alternativas a la red de distribución y trasladarla a sus destinos. Por consiguiente, se necesitan esfuerzos coordinados para adaptar la energía solar fotovoltaica y la energía eólica a los nuevos dispositivos en los sistemas de las redes inteligentes, por lo que estos últimos deben ser capaces de integrarse con los equipos existentes y disponer de herramientas informáticas para analizar la integración de las energías renovables en los sistemas energéticos. Estas herramientas energéticas son diversas en términos de aplicaciones, tecnologías correspondientes y objetivos que realizan. Grzonka et al. (2015).

Los estudios de factibilidad y viabilidad en todo el mundo son comunes. Estos pueden ser utilizados como insumos para el desarrollo de varios sistemas de energía renovable conectados a la red para localidades específicas. Por ejemplo, una investigación de $80 \mathrm{~kW}$ de energía solar fotovoltaica conectado al sistema, utilizando el software de optimización de energía HOMER, demostró la viabilidad del sistema que se describe en Grzonka et al. (2015). De esta marea, la investigación detalla una estrategia operativa aplicada en isla que confirma que un sistema eléctrico confiable y eficiente es alcanzable usando una combinación de unidades eólicas e hidroeléctricas. Otro ejemplo que se tiene, es presentado en Merino et al. (2012), en donde se desarrolló un modelo de análisis de estabilidad transitoria para una pequeña red aislada cuyo suministro energético principalmente está dado por fuentes renovables. Por otro lado, la optimización de siete aldeas remotas del distrito de Almora, estado de Uttarakhand, la India, detalla el escenario más confiable y rentable (mezcla de fuentes renovables) para el área.

\section{MICROGRIDS Y PROYECTOS}

En muchos países se están llevando a cabo MG inteligentes como proyectos piloto relacionados. Igualmente el intercambio de conocimientos y experiencias ha allanado el camino para una mejor planificación y ejecución de los proyectos de las SG. Es así como países tales como: Australia, Canadá, Gran Bretaña, Estados Unidos, Corea del Sur, Irlanda, Colombia y Japón, están fomentando la estrategia gubernamental para lograr la seguridad energética y las bajas emisiones de carbono en el desarrollo de las fuentes no convencionales de suministro eléctrico. En consecuencia, el Informe de la federación global de redes inteligentes de 2012 (Hiscock y Beauvais, 2013) ha señalado lo siguiente: El proyecto comunidades de redes inteligentes en Australia está probando la detección de fallas en la red, el aislamiento y la restauración, el monitoreo de la calidad de la potencia eléctrica y la automatización de la distribución con un sistema de administración de distribución comercial. Por consiguiente, la unión de fuerzas en la implementación de fuentes convencionales del suministro en diferentes países permitirá tener la revolución energética que tanto espera la humanidad.

Bajo la anterior reflexión de la federación global, también cabe destacar a Canadá, cuya clasificación dinámica propende a optimizar la capacidad de transferencia de la transmisión en la zona ancha de sistema de control, mejorando así la estabilidad del voltaje, y la iniciativa de medición inteligente de Ontario, que logró una reducción de los picos de $5 \%$ al $8 \%$ en el perfil de carga de los usuarios. Por otro lado, el 
programa "Londres bajo en carbono" integró una serie de tecnologías de baja emisión en donde instalaciones fotovoltaicas, medidores inteligentes, vehículos eléctricos, estaciones de carga y bombas de calor en la red de distribución mejoran considerablemente la eficiencia energética y la respuesta a la demanda. Así pues, se ha integrado en la red nacional la primera solución de almacenamiento de energía criogénica (líquido a baja temperatura) del mundo, de allí, el nombre de Piloto de almacenamiento de energía criogénica. Igualmente, en USA, el proyecto de demonstración de redes inteligentes del noroeste del pacífico, muestra un proyecto piloto que abarca cinco estados (Montana, Washington, Idaho, Oregón y Wyoming) e incluye 22 servicios públicos para demostrar la coordinación continua de activos de SG. Seguidamente, Houston implementa un sistema de medición totalmente integrado, un portal web para el cliente y una notificación de interrupción automática. Además, Smart Texas implementa un despliegue masivo de medidores inteligentes y automatización de distribución.

En semejanza a los anteriormente expuestos, Corea tiene la famosa Jeju SG Demonstration Complex incorporando tecnologías en energía eólica y energía solar fotovoltaica, vehículos eléctricos, IMA, almacenamiento de energía, automatización distribuida, monitoreo de red y telemetría. Allí, el programa Transporte inteligente implementa una infraestructura de VE que depende de la comunicación inalámbrica. También, el Sistema operativo de fuente de energía renovable es una demostración de la MG que incorpora tecnologías de generación y generación de energía eólica de gran volumen. El Smart Place, que participa en el consumidor, introduce tarifas de electricidad en tiempo real, fuentes de energía renovables, electrodomésticos inteligentes y soluciones de almacenamiento con pantallas en el hogar. Por otro lado, en Irlanda, la Comisión de Regulación de la Energía (CER) ha implementado un ensayo de medidores inteligentes en alrededor de 9000 hogares y en empresas del sector público y privado. De tal modo que el proyecto Ilamado Ecar Ireland como piloto en una infraestructura de carga de VE, permite que los conductores de los vehículos paguen al proveedor de electricidad y no la estación de carga, lo cual representa un gran despliegue de comunicaciones para garantizar un sistema altamente operado y controlado.

Finalmente, el proyecto europeo de Integración de Energías Renovables en la Red Europea de Electricidad (IGREENGrid) utiliza un verdadero despliegue a gran escala de IMA (López et al. 2015), centrado principalmente en aumentar la capacidad de alojamiento de fuentes de energía renovable en las redes de distribución, sin comprometer la confiabilidad y la calidad del suministro. Este proyecto despliega más de 200.000 medidores inteligentes en la zona de Madrid. Lo cual permite dimensionar un panorama de los datos de campo necesarios para preparar la reglamentación que debe promover la innovación de los sistemas de distribución para las nuevas redes inteligentes.

\section{INVESTIGACIÓN FUTURA Y DESAFÍOS EN LAS REDES INTELIGENTES}

La investigación de SG ha recorrido un largo camino desde que salió por primera vez en 1997. Si bien muchas tecnologías, sistemas, dispositivos, métodos y procesos que mejoran las redes inteligentes han surgido, existe un inmenso potencial para futuras investigaciones. Entre ellos están: nuevos métodos de predicción de series de tiempo para SGs; nuevas infraestructuras de comunicaciones para redes de autosanación, estudios de fiabilidad y calidad energética mejorados; mejora en optimización de flujo de potencia; nuevas técnicas de diseño en baterías para VE a fin de prolongar su vida útil. Así como el desarrollo de controles basados en la información de la nube y los estudios de gestión (Mital et al. 2015).

A pesar del obvio éxito en el desarrollo de la SG, en sus tecnologías y sistemas, los problemas y desafíos los rodean, y el éxito final sigue estando bastante lejos. Los siguientes temas y desafíos son también áreas de investigación potenciales: tecnologías V2G: desgaste de la batería y nuevas tecnologías de almacenamiento. Igualmente, barreras para la implementación: costo y beneficio, conocimiento, inercia institucional, participación de los consumidores, protección de datos, privacidad, problemas de seguridad y desafíos en la SG, diseño de enrutamiento, interoperabilidad y conformidad con las normas, seguridad física, seguridad cibernética, vulnerabilidades en AMI, Integración de protocolos en forma transparente, mejora de la eficiencia energética en dispositivos de comunicaciones, mejora en el almacenamiento de energía, modernización de la red, estudio de franjas horarias, mejoras en la dinámica de redes, realización de algoritmos multiobjetivos. Así como simuladores y co-simuladores de SG capaces de simular escenarios sencillos, sincronización de tiempo e intercambio de datos; problema de compatibilidad con dispositivos inteligentes, normas tecnológicas para el registro de datos y requisitos de comunicación; generación distribuida en sistemas de distribución y automatización de sistemas de distribución (Muttaqi et al. 2015); el diseño de esquemas de cifrado de datos fuertes.

\section{CONCLUSIONES}

El concepto de SG ha evolucionado de una visión a una meta que se está realizando lentamente. A medida que la tecnología ha crecido, los dispositivos y sistemas son capaces de soportar la formación de una red 
más inteligente. Por consiguiente, las políticas energéticas concretas están facilitando las iniciativas de SG para todas las naciones. Lo anterior en aras de obtener sistemas robustos que faciliten la mejorara en la confiabilidad del suministro, la calidad de la potencia eléctrica y la eficiencia energética.

Con base en lo anterior, existen importantes oportunidades de investigación en las áreas de previsión de series de tiempo en SGs, estudios de fiabilidad y calidad de energía, optimización de flujo de energía, sistemas de baterías, computación en nube de información y la integración de prácticas de fuentes de energía renovable a gran escala. Incluso los problemas y desafíos identificados como el uso de baterías en V2G, la protección de datos, la seguridad física y cibernética, las limitaciones de los simuladores y la automatización del sistema de distribución pueden ser buenos puntos de partida para futuras investigaciones.

Por último, la idea básica de la SG no es suficiente al embarcarse en este complejo sistema, incluso con las experiencias y las tecnologías que están disponibles, la búsqueda de la red ideal es una inversión en función del tiempo, del dinero y de la investigación y de las pruebas continuas. Con los grandes esfuerzos puestos adelante para la investigación de las SG, puede ser más eficaz para ayudar a lograr la sostenibilidad energética y la conservación y preservación ambiental. El futuro exacto de la SG puede ser difícil de predecir, pero las innovaciones recientes muestran una fusión dinámica de los agentes del sector eléctrico en aras de realizar la revolución energética que tanto anhela la humanidad.

\section{AGRADECIMIENTOS}

Los autores de este artículo desean agradecer a Colciencias y a la Universidad Distrital Francisco José de Caldas por la financiación de los recursos para el desarrollo de este proyecto de investigación.

\section{REFERENCIAS}

Ashok, A.; A. Hahn y M. Govindarasu, Cyber-physical security of Wide-Area Monitoring, Protection and Control in a smart grid environment. Journal of Advanced Research, 5(4), 481-489 (2014).

Avelar, E.; L. Marques; D. Passos; R. Macedo; K. Dias y M. Nogueira, Interoperability issues on heterogeneous wireless communication for smart cities. Computer Communications, 58, 4-15 (2015).

Bekara, C., Security issues and challenges for the iot-based smart grid. Procedia Computer Science, 34, 532-537 (2014).

Berizzi, A.; C. Bovo; V. Llea; M. Merlo; A. Miotti y F. Zanellini, Decentralized congestion mitigation in HV distribution grids with large penetration of renewable generation. International Journal of Electrical Power \& Energy Systems, 71, 51-59 (2015).

Bhor, D.; K. Angappan y K. M. Sivalingam, Network and power-grid co-simulation framework for Smart Grid wide-area monitoring networks. Journal of Network and Computer Applications, 59, 274-284 (2016).

Birk, M. E., Impact of distributed energy resources on locational marginal prices and electricity networks. Massachusetts Institute of Technology (2016).

Construyored, Eolo, El Primer Auto Eólico 100\% Colombiano, Retrieved from (en linea: https://construyored.com/noticias/1274, acceso: 10 de marzo 2017), Construyored en Colombia (2017).

Di Tommaso, A. O.; S. Favuzza; F. Genduso; R. Miceli, y G. R. Galluzzo, Development of diagnostic systems for the fault tolerant operation of Micro-Grids. In Power Electronics Electrical Drives Automation and Motion (SPEEDAM), 2010 International Symposium on (pp. 1645-1650). IEEE (2010).

Doguc, O. y J. Ramirez-Marquez, An automated method for estimating reliability of grid systems using Bayesian networks. Reliability Engineering \& System Safety, 104, 96-105 (2012).

Farag, M. M., M. Azab y B. Mokhtar, Cross-layer security framework for smart grid: Physical security layer. In Innovative Smart Grid Technologies Conference Europe (ISGT-Europe), 2014 IEEE PES (pp. 1-7). IEEE (2014).

Grzonka, D.; J. Kołodziej; J. Tao y S. U. Khan, Artificial Neural Network support to monitoring of the evolutionary driven security aware scheduling in computational distributed environments. Future Generation Computer Systems, 51, 72-86 (2015).

Hernandez, C.; V. Gomez y A. Pena, Calidad del servicio en la energía eléctrica residencial. equipo medidor de indicadores des y fes, primera edición, 24-36. Editorial UD, Bogota, Colombia (2014).

Hiscock, J. y D. Beauvais, Smart grid in Canada 2012-2013. report (2013).

Jarmakiewicz, J.; K. Parobczak y K. Maślanka, Cybersecurity protection for power grid control infrastructures. International Journal of Critical Infrastructure Protection, 18, 20-33 (2017).

Kaschel Cárcamo, H. y E. Viera Riquelme, Desarrollo de protocolo de comunicaciones para redes HAN en SmartGrids. Ingeniare. Revista Chilena de Ingeniería, 25(1), 15-27 (2017). 
Kilkki, O.; A. Kangasrääsiö; R. Nikkilä; A. Alahäivälä y I. Seilonen, Agent-based modeling and simulation of a smart grid: a case study of communication effects on frequency control. Engineering Applications of Artificial Intelligence, 33, 91-98 (2014).

Kobus, C. B. A.; E. A. M. Klaassen; R. Mugge y J. P. L. Schoormans, A real-life assessment on the effect of smart appliances for shifting households' electricity demand. Applied Energy, 147, 335-343 (2015).

Kuzlu, M.; M. Pipattanasomporn y S. Rahman, Communication network requirements for major smart grid applications in HAN, NAN and WAN. Computer Networks, 67, 74-88 (2014).

Liu, T.; Y. Sun; Y. Liu; Y. Gui; Y. Zhao; D. Wang y C. Shen, Abnormal traffic-indexed state estimation: A cyber-physical fusion approach for smart grid attack detection. Future Generation Computer Systems, 49, 94-103 (2015).

López, G.; J. I. Moreno; H. Amarís y F. Salazar, Paving the road toward smart grids through large-scale advanced metering infrastructures. Electric Power Systems Research, 120, 194-205 (2015).

Lucas, A.; C. A. Silva y R. C. Neto, Life cycle analysis of energy supply infrastructure for conventional and electric vehicles. Energy Policy, 41, 537-547 (2012).

Mah, D. N.; J. M. Van Der Vleuten; J. C. Ip y P. R. Hills, Governing the transition of socio-technical systems: a case study of the development of smart grids in Korea. Energy Policy, 45, 133-141 (2012).

Meddeb, A., Internet of things standards: who stands out from the crowd? IEEE Communications Magazine, 54(7), 4047 (2016).

Merino, J.; C. Veganzones, J. A. Sanchez; S. Martinez y C. A. Platero, Power system stability of a small sized isolated network supplied by a combined wind-pumped storage generation system: A case study in the Canary Islands. Energies, 5(7), 2351-2369 (2012).

Mital, M.; A. K. Pani; S. Damodaran y R. Ramesh, Cloud based management and control system for smart communities: A practical case study. Computers in Industry, 74, 162-172 (2015).

Muttaqi, K. M.; J. Aghaei; V. Ganapathy y A. E. Nezhad, Technical challenges for electric power industries with implementation of distribution system automation in smart grids. Renewable and Sustainable Energy Reviews, 46, 129142 (2015).Nistor, S.; J. Wu; M. Sooriyabandara y J. Ekanayake, Capability of smart appliances to provide reserve services. Applied Energy, 138, 590-597 (2015).

Nunes, P.; T. Farias y M. C. Brito, Enabling solar electricity with electric vehicles smart charging. Energy, 87, 10-20 (2015).

Peng, L. y G.-S. Yan, Clean energy grid-connected technology based on smart grid. Energy Procedia, 12, 213218 (2011).

Penya, Y. K.; J. C. Nieves; A. Espinoza; C. E. Borges; A. Pena y M. Ortega, Distributed semantic architecture for smart grids. Energies, 5(11), 4824-4843 (2012).

R. Abby, Applying Lessons Learned from One of the Biggest Blackouts in History. Foros en la web, (en linea en: goo.gl/WPC98w, acceso: 19 de marzo 2017), (2014).

Rahimi, F. y A. Ipakchi, Demand response as a market resource under the smart grid paradigm. IEEE Transactions on Smart Grid, 1(1), 82-88 (2010).

Reddy, K. S.; M. Kumar; T. K. Mallick; H. Sharon y S. A. Lokeswaran, review of Integration, Control, Communication and Metering (ICCM) of renewable energy based smart grid. Renewable and Sustainable Energy Reviews, 38, 180-192 (2014).

Restrepo-Garcés, A. R.; D. F. Manotas-Duque y C. A. Lozano, Portafolio para Auto-Generación de Electricidad con Fuentes Renovables en Edificios Comerciales. Información Tecnológica, 27(1), 91-104 (2016).

Schneider Electric, Foro sobre EcoStruxure y nuevas tecnologías, (en linea: https://goo.gl/vxuGSy, acceso: 16 de Julio de 2017) (2017).

Singh, M. y U. Grape, Office of Electricity Delivery and Energy Reliability (OE) National Energy Technology Laboratory (NETL) American Recovery and Reinvestment Act 2009 United States Department of Energy. Seeo, Incorporated, Hayward, CA (United States) (2014).

Singh, V. P.; N. Kishor y P. Samuel, Distributed Multi-Agent System-Based Load Frequency Control for Multi-Area Power System in Smart Grid. IEEE Transactions on Industrial Electronics, 64(6), 5151-5160 (2017).

Stastny, L.; L. Franek y Z. Bradac, Time synchronized low-voltage measurements for Smart Grids. Procedia Engineering, 100, 1389-1395 (2015).

Suleiman, H.; I. Alqassem; A. Diabat; E. Arnautovic y D. Svetinovic, Integrated smart grid systems security threat model. Information Systems, 53, 147-160 (2015).

Tarhuni, N. G.; N. I. Elkalashy; T. A. Kawady y M. Lehtonen, Autonomous control strategy for fault management in distribution networks. Electric Power Systems Research, 121, 252-259 (2015).

Tsado, Y.; D. Lund y K. A. A. Gamage, Resilient communication for smart grid ubiquitous sensor network: State of the art and prospects for next generation. Computer Communications, 71, 34-49 (2015). 
Wu, Y.; J. Chen y L. Liu, Construction of China's smart grid information system analysis. Renewable and Sustainable Energy Reviews, 15(9), 4236-4241 (2011).

Xia, S.; X. Luo y K. W. Chan, framework for self-healing smart grid with incorporation of multi-agents. Energy Procedia, $61,2123-2126$ (2014).

Yilmaz, M. y P. T. Krein, Review of benefits and challenges of vehicle-to-grid technology. In Energy Conversion Congress and Exposition (ECCE), 2012 IEEE (pp. 3082-3089). IEEE (2012).

Yu, Y.; J. Yang y B. Chen, The smart grids in China-A review. Energies, 5(5), 1321-1338 (2012).

Yuan, Y.; Z. Wei; G. Sun; Y. Sun y D. A. Wang, real-time optimal generation cost control method for virtual power plant. Neurocomputing, 143, 322-330 (2014). 
